\title{
Effectiveness and Characteristics of Acupressure for Elderly with Insomnia: A Systematical Review
}

\author{
Zidan Gong and Winnie Yu* \\ Institute of Textiles and Clothing, The Hong Kong Polytechnic University, Hong Kong
}

Submission: July 10, 2017; Published: July 28, 2017

*Corresponding author: Winnie Yu, Institute of Textiles and Clothing, The Hong Kong Polytechnic University, Hong Kong,

Email: winnie.yu.itc@polyu.edu.hk

\begin{abstract}
The symptom of insomnia is one of the most common complaints among the elderly during their aging process. It is defined as the subjective reported insufficient quality or quantity of sleep despite adequate sleeping conditions. The prevalence of insomnia could reach $40 \%-50 \%$ among the elderly and significantly affect their quality of life. This paper aims to conduct systematic review on previous studies to evaluate the effectiveness and summarize the treatment characteristics of acupressure. Limitations and recommendation were provided as future directions of therapeutic garments development for the elderly. This new concept of acupressure therapeutic garment could help to identify the future development directions of therapeutic function garments and provide promising methods to improve the insomnia and life quality of the elderly.
\end{abstract}

Keywords: Insomnia; Elderly; Therapeutic garment; Acupressure

\section{Introduction}

The aging process is universal and biological that everyone must experience. Both psychological and physical changes may occur when people becoming old and the symptom of insomnia is one of the most common complaints among the elderly [1,2]. Insomnia is defined as the subjective reported insufficient quality or quantity of sleep despite adequate sleeping conditions $[3,4]$. Prevalence of this symptom increases along with age resulting that about $40 \%-50 \%$ of the elderly suffering from insomnia $[1,5,6]$.

Insomnia among elderly may have many negative influences on their general health and life quality. Specifically, insomnia could cause daytime fatigue and malaise, poor concentration, memory loss, cognitive decline, mood disturbances, daytime sleepiness or other symptoms such as headaches, hypertension and gastrointestinal problems [3,5-8]. Moreover, the statewide research conducted by the Michigan Nursing Homes indicated that the ignorance of insomnia could highly lead to the risk of falls (increased by 50\%) which may cause a higher rate of mortality among the elderly [6,9]. However, many people in the public including some doctors have the misconception that the symptom of insomnia among elderly is normal and accepted as the process of aging. Actually, the insomnia was caused by both medical and psychosocial factors as co morbidities [10]. To summarize, the common causes of insomnia among elderly are: i. Physical symptoms, such as chronic low back pain, joint diseases etc.

ii. Underlying medical condition or side effect of medication.

iii. Mental diseases, such as anxiety or depression.

iv. Environmental or behavioral factors. [3,6,11,12].

To treat for the insomnia among elderly, treatments could be classified into Pharmacological and Nonphamacologic therapy. Pharmacological treatment includes some drugs such as Benzodiazepine sedative hypnotics, Non-benzodiazepine sedative hypnotics, Sedative antidepressants which could make people calm down and feel sleepy. Non phamacologic therapy refers to Bright light therapy, Cognitive behavior therapy, Stimulus control therapy, Sleep Hygiene, etc. $[3,4,13]$.

However, although there are numerous normal and tradition treatments for insomnia, there still remain limitations since the group of elderly is special. Most of the cases, elderly have a rich medical history. They not only suffered from the symptom of insomnia but also have other diseases such as heart diseases and hypertension. Generally, the health condition of the elderly is weaker than younger adults. If receiving pharmacological treatment, side effect could be a big issue especially when there is a drug interaction because many elderly take other medication 
for different diseases [14]. Then if non pharmacological treatment adopted, corresponding conditions including professional devices, room for treatment, consultants, etc. are required which cost much time and energy and cause much inconvenience. Thus, the alternative therapy of acupressure will be suitable for treating insomnia in the elderly. Acupressure belongs to the Traditional Chinese Medicine (TCM) as component of alternative treatments [15]. It was defined as a technique which applies pressure on acupoints on human body by using finger, palms or other devices to balance the vital energy flows called Qi through meridians $[7,16,17]$. This traditional treatment is gaining increasing popularity due to its non-invasive, non-side effect and home treatment nature. This paper specifically focus on the elderly with insomnia while consider the acupressure therapy. A systematical review was conducted to evaluate the effectiveness of acupressure and summarize the treatment characteristic as a preliminary study of acupressure therapeutic apparel development for the elderly with insomnia.

\section{Methods}

\section{Data sources and searches}

To start with systematic review, a literature search was conducted in March 2016 initially focusing on any available published research papers about acupressure for insomnia among elderly. Five databases including Medline, Science Direct, CINAHL, Cochrane Library, Chinese Academic Journals were accessed for paper searching using the Keywords: acupressure, acupoints, traditional Chinese massage, insomnia, sleep quality, sleep disorders, sleep disturbance, elderly, old people, silver group, aging people, senior citizen. Besides, additional publications were identified through other sources or hand searching.

\section{Exclusion criteria}

A large number of research articles were collected for first and second scans. Articles which are not quite related to acupressure for elderly with insomnia must be excluded to simplify further analysis. Exclusion criteria are set up referring these points below: i. Duplications

ii. Randomized and controlled design: articles do not have a randomized controlled trial will be excluded

iii. Sample size: less than 30 will be excluded

iv. Acupoints: articles have not noted specific acupressure points on human body will be excluded

v. Unsuitable symptoms: except insomnia, samples in research studies have other serious diseases such as cancer, stroke, Alzheimer, etc. should be excluded

vi. Unsuitable sample: studies focused on pregnant women, adolescents, bed-ridden elderly, psychiatric patients, etc. but not active elderly were excluded

vii. Unsuitable treatment: researches used needling acupuncture or used acupressure as an assistant of other therapies should be excluded

viii. Others: articles which are too general or conduct research unconventional or unorthodox, etc. will be excluded

\section{Assessment of risk of bias}

Two evaluators (Gong and Ameersing) independently assessed the quality of selected trials according to the modified Jada Scale [18]. There are five questions set up in the scoring system:

a. Described as randomized?

b. Appropriate randomization method described?

c. Intervention blinded to the subject?

d. Intervention blinded to the evaluator?

e. Description of withdrawals and dropouts?

Answer to each question should be a "Yes" or a "No" matching the score of 1 point or 0 point. Score of the modified Jada scale could range from 0 to 5 that higher score indicates lower risk of bias and higher quality of the trial.

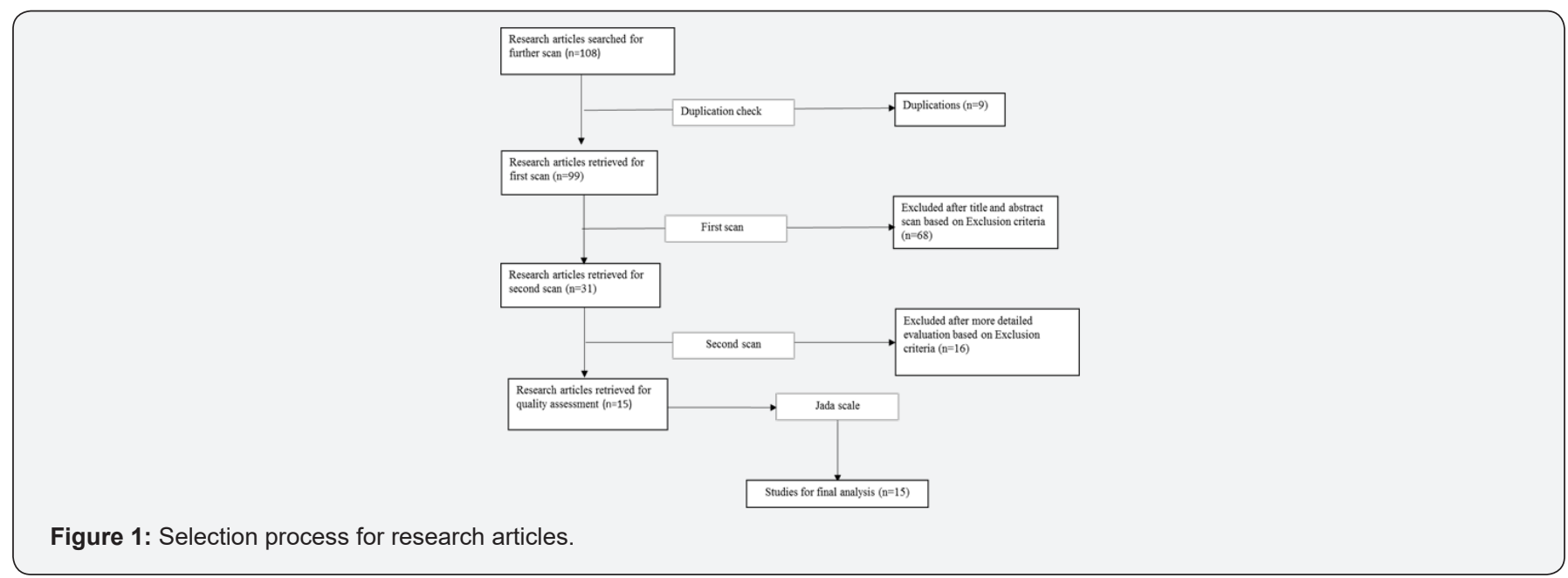




\section{Results and Discussion}

The literature search initially find 108 potential research articles for further scan based on the selection process in Figure 1. Specifically, 9 articles were excluded after the duplication check. Then the first san was conducted among the remaining 99 studies by reviewing the titles and abstracts followed by the detailed evaluation of the second scan. Totally, there are 93 articles excluded in the whole scan process according to various exclusion criteria. Finally, 15clinical trial papers ( 3 from PubMed, 2 from Cochrane Library, 3 from Chinese Academic Journals, 4 from Science Direct, 2 from CINAHL and 1 from other sources) are remained for further analysis.

\section{Quality assessment}

Table 1: Jada score of two independent evaluator.

\begin{tabular}{|c|c|c|c|c|c|c|c|c|c|c|c|c|c|c|c|c|c|c|c|c|c|c|c|c|c|c|c|c|c|c|}
\hline $\begin{array}{c}\text { Items /paper } \\
\text { No. }\end{array}$ & 1 & & 2 & & 3 & & 4 & & 5 & & 6 & & 7 & & 8 & & 9 & & 10 & & 11 & & 12 & & 13 & & 14 & & & 15 \\
\hline Evaluator & E1 & E2 & E1 & E2 & E1 & E2 & E1 & E2 & E1 & E2 & E1 & E2 & E1 & E2 & E1 & E2 & E1 & E2 & E1 & E2 & E1 & E2 & E1 & E2 & E1 & E2 & E1 & E2 & E1 & E2 \\
\hline $\begin{array}{l}\text { Described as } \\
\text { randomized }\end{array}$ & 1 & 1 & 1 & 1 & 1 & 1 & 1 & 1 & 1 & 1 & 1 & 1 & 1 & 1 & 1 & 1 & 1 & 1 & 1 & 1 & 1 & 1 & 1 & 1 & 1 & 1 & 1 & 1 & 1 & 1 \\
\hline $\begin{array}{l}\text { Appropriate } \\
\text { randomization } \\
\text { method } \\
\text { described }\end{array}$ & 1 & 1 & 1 & 1 & 1 & 1 & 1 & 1 & 1 & 1 & 1 & 1 & 1 & 1 & 1 & 1 & 1 & 1 & 1 & 1 & 0 & 0 & 1 & 0 & 0 & 0 & 1 & 1 & 1 & 1 \\
\hline $\begin{array}{c}\text { Intervention } \\
\text { blinded to the } \\
\text { subject }\end{array}$ & 1 & 1 & 1 & 1 & 0 & 0 & 1 & 1 & 1 & 1 & 1 & 1 & 1 & 1 & 0 & 0 & 1 & 1 & 1 & 0 & 0 & 0 & 0 & 0 & 0 & 0 & 0 & 0 & 0 & 0 \\
\hline $\begin{array}{c}\text { Intervention } \\
\text { blinded to the } \\
\text { evaluator }\end{array}$ & 0 & 0 & 1 & 1 & 0 & 0 & 1 & 1 & 1 & 1 & 1 & 1 & 1 & 1 & 0 & 0 & 1 & 1 & 1 & 0 & 0 & 0 & 0 & 0 & 0 & 0 & 1 & 0 & 0 & 0 \\
\hline Total & 4 & 4 & 5 & 5 & 3 & 3 & 5 & 5 & 5 & 5 & 5 & 5 & 5 & 5 & 3 & 3 & 5 & 5 & 5 & 3 & 1 & 1 & 2 & 1 & 1 & 1 & 4 & 3 & 3 & 3 \\
\hline
\end{tabular}

Note: E1, evaluator 1; E2, evaluator 2

All of these 15 studies were reported as randomized clinical trials. The detailed scoring result of the modified Jada scale given by two independent evaluators is presented in the Table 1attached at the end. $80 \%$ of the reviewed studies had the Jada score $\geq 3$ that indicate a good quality with a relatively low risk of bias. Moreover, 6 of these fifteen trails (study 2, 4, 5, 6, 7, 9) have got the full mark of 5 from both evaluators. Only 3 trails Table 2: Basic information of 15 trails. (study 11, 12, 13) got the Jada score below 2 without blinded trail design and dropout description. However, these 3 studies were all written in Chinese by experts or doctors and conducted in hospitals or nursing home that still have good referent value. The score variation may due to the theory difference between Chinese and Western medicine.

\begin{tabular}{|c|c|c|c|c|c|c|c|}
\hline No. & Author (year) & Sample size & Average age & Treatment protocol & Measures & Effectiveness & Sources \\
\hline 1 & $\begin{array}{l}\text { Chen et al. } \\
\text { [24] }\end{array}$ & 84 & $\begin{array}{c}79.04 \\
\text { years old }\end{array}$ & $\begin{array}{l}\text {-Acupressure group received } \\
\text { massage on acupoints. } \\
\text {-Sham acupressure group } \\
\text { received massage 1-3 cun } \\
\text { away from acupoints. } \\
\text {-Control group received only } \\
\text { conversation }\end{array}$ & - PSQI & $\begin{array}{l}\text { Acupressure group } \\
\text { >sham group } \\
\text { No significant } \\
\text { difference between } \\
\text { sham and control } \\
\text { group }\end{array}$ & CINAHL \\
\hline 2 & $\begin{array}{c}\text { Nesami et al. } \\
\text { [27] }\end{array}$ & 90 & $\begin{array}{c}60.3 \\
\text { years old }\end{array}$ & $\begin{array}{l}\text {-Acupressure group apply } \\
\text { acupressure on acpoints for } \\
18 \text { min. } \\
\text {-Valerian acupressure group } \\
\text { receive acupressure with } \\
\text { two drops of valerian oil }\end{array}$ & - SMHSQ & $\begin{array}{l}\text { Both acupressure } \\
\text { and valerian } \\
\text { acupressure group } \\
\text { >control group } \\
\text { No significant } \\
\text { difference between } \\
\text { these two groups. }\end{array}$ & $\begin{array}{c}\text { Science } \\
\text { Direct }\end{array}$ \\
\hline
\end{tabular}




\begin{tabular}{|c|c|c|c|c|c|c|c|}
\hline & & & & $\begin{array}{l}\text {-Control group received } \\
\text { massage at points that were } \\
\text { away from the main points } \\
\text { using the same technique } \\
\text { and for the same length of } \\
\text { time }\end{array}$ & & & \\
\hline 3 & $\begin{array}{l}\text { Lei et al. } \\
\text { [26] }\end{array}$ & 68 & $\begin{array}{c}71.3 \\
\text { Years old }\end{array}$ & $\begin{array}{l}\text {-Treatment group received } \\
\text { acupoint massage on the } \\
\text { base of the interventions } \\
\text { given to the control group } \\
\text {-Control group reveived } \\
\text { routine psychological } \\
\text { health guidance and sleep } \\
\text { knowledge education }\end{array}$ & $\begin{array}{l}\text { - PSQI } \\
\text { - MMSE }\end{array}$ & $\begin{array}{l}\text { Treatment group } \\
>\text { control group }\end{array}$ & $\begin{array}{l}\text { Chinese } \\
\text { Academic } \\
\text { Journals }\end{array}$ \\
\hline 4 & $\begin{array}{c}\text { Shariati et al. } \\
\text { [28] }\end{array}$ & 48 & $\begin{array}{c}54.5 \\
\text { Years old }\end{array}$ & $\begin{array}{l}\text {-Acupressure group received } \\
\text { acupressure plus routine } \\
\text { care } \\
\text {-Control group received only } \\
\text { routine care }\end{array}$ & -PSQI & $\begin{array}{l}\text { Acupressure Group } \\
>\text { Placebo group }\end{array}$ & $\begin{array}{l}\text { Science } \\
\text { Direct }\end{array}$ \\
\hline 5 & $\begin{array}{c}\text { Nordio \& } \\
\text { Romanelli } \\
\text { [19] }\end{array}$ & 40 & $\begin{array}{c}64.05 \\
\text { years old }\end{array}$ & $\begin{array}{l}\text {-Acupressure group received } \\
\text { wrist acupressure device } \\
\text { treatment on acupoint } \\
\text {-Placebo group received } \\
\text { wrist acupressure treatment } \\
\text { on non-acupoint. }\end{array}$ & $\begin{array}{l}\text {-GHQ } \\
\text {-STAI } \\
\text { - PSQI } \\
\text { - MLT }\end{array}$ & $\begin{array}{c}\text { Acupressure } \\
\text { treatment }> \\
\text { Control group }\end{array}$ & PubMed \\
\hline 6 & $\begin{array}{l}\text { Tsay } \\
{[21]}\end{array}$ & 98 & 55.5 years old & $\begin{array}{c}\text {-Experimental receive } \\
\text { acupressureplus usual care } \\
\text {-Placebo receive sham } \\
\text { acupressure } 1 \mathrm{~cm} \text { away } \\
\text { meridians plus usual care } \\
\text {-Controlgroups receive } \\
\text { usual care }\end{array}$ & $\begin{array}{c}\text {-PSQI } \\
\text {-Sleep Log } \\
\text {-MOS SF-36 }\end{array}$ & $\begin{array}{l}\text { Acupressure group } \\
>\text { Sham group }> \\
\text { Control group }\end{array}$ & CINAHL \\
\hline 7 & $\begin{array}{c}\text { Abedian et al. } \\
\text { [15] }\end{array}$ & 120 & $\begin{array}{c}50.7 \\
\text { Years old }\end{array}$ & $\begin{array}{l}\text {-Acupressure group received } \\
\text { massage on acupoints. } \\
\text {-Sham acupressure group } \\
\text { received massage on non- } \\
\text { acupints 1-3 cun away from } \\
\text { true points. } \\
\text {-The control group only } \\
\text { received the weekly control } \\
\text { of blood pressure and } \\
\text { speech communication } \\
\text { about health question. }\end{array}$ & - PSQI & $\begin{array}{l}\text { Acupressure group } \\
>\text { Sham group }> \\
\text { Control group }\end{array}$ & PubMed \\
\hline 8 & $\begin{array}{l}\text { Zheng et al. } \\
\text { [32] }\end{array}$ & 75 & $\begin{array}{c}59.84 \\
\text { years old }\end{array}$ & $\begin{array}{l}\text {-Experiental group received } \\
\text { both conventional treatment } \\
\text { \& health guidance and } \\
\text { acupressure treatment. } \\
\text {-Control group only receive } \\
\text { conventional treatment \& } \\
\text { health guidance }\end{array}$ & $\begin{array}{c}\text {-PSQI } \\
\text {-Blood pressure }\end{array}$ & $\begin{array}{l}\text { Experimental } \\
\text { group }>\text { control } \\
\text { group }\end{array}$ & $\begin{array}{l}\text { Cochrane } \\
\text { Library }\end{array}$ \\
\hline 9 & $\begin{array}{l}\text { Reza, et al. } \\
\text { [7] }\end{array}$ & 90 & $\begin{array}{c}75.21 \\
\text { years old }\end{array}$ & $\begin{array}{c}\text {-Acupressure group received } \\
\text { acupressure plus usual care. } \\
\text {-Sham acupressure group } \\
\text { received sham acupressure } \\
0.5 \text { cun away from true } \\
\text { acupressure points and } \\
\text { usual cure. } \\
\text {-Control group received } \\
\text { usual cure }\end{array}$ & $\begin{array}{c}\text {-PSQI } \\
\text {-Sleep log }\end{array}$ & $\begin{array}{c}\text { Acupressure group } \\
\text { >Sham group } \\
\text { Control group } \\
\text { No significant } \\
\text { difference between } \\
\text { sham and control } \\
\text { group }\end{array}$ & $\begin{array}{l}\text { Science } \\
\text { Direct }\end{array}$ \\
\hline
\end{tabular}




\begin{tabular}{|c|c|c|c|c|c|c|c|}
\hline 10 & $\begin{array}{c}\text { Sun et al. } \\
\text { [29] }\end{array}$ & 50 & $\begin{array}{c}68.83 \\
\text { years old }\end{array}$ & $\begin{array}{l}\text {-Acupressure group reveived } \\
\text { standard acupressure on the } \\
\text { HT7 points of both wrists. } \\
\text {-Control group received } \\
\text { only light touch on the same } \\
\text { places }\end{array}$ & $\begin{array}{l}\text { - AIS } \\
\text { - PSQI }\end{array}$ & $\begin{array}{l}\text { Experimental } \\
\text { group > control } \\
\text { group }\end{array}$ & $\begin{array}{l}\text { Science } \\
\text { Direct }\end{array}$ \\
\hline 11 & $\begin{array}{c}\text { Qiu et al. } \\
\text { [20] }\end{array}$ & 89 & $\begin{array}{c}46 \\
\text { years old }\end{array}$ & $\begin{array}{l}\text {-Treatment group received } \\
\text { acupressure at night } \\
\text {-Control group eat } \\
\text { Estazolam 2mg every night. }\end{array}$ & $\begin{array}{c}\text {-Sleeping } \\
\text { duration time }\end{array}$ & $\begin{array}{c}\text { Total effective rate } \\
\text { of treatment group } \\
\text { is } 92.5 \% \text {. } \\
\text { Total effective rate } \\
\text { of control is } 86.1 \% \text {. }\end{array}$ & $\begin{array}{c}\text { Chinese } \\
\text { Academic } \\
\text { Journals }\end{array}$ \\
\hline 12 & $\begin{array}{c}\text { He et al. } \\
\text { [24] }\end{array}$ & 66 & $\begin{array}{c}72 \\
\text { years old }\end{array}$ & $\begin{array}{l}\text {-Treatment group received } \\
\text { acupressure at night } \\
\text {-Control group eat } \\
\text { Estazolam 2mg every night. }\end{array}$ & $\begin{array}{c}\text {-Sleeping } \\
\text { duration time }\end{array}$ & $\begin{array}{c}\text { Total effective rate } \\
\text { of treatment group } \\
\text { is } 93.9 \% \text {. } \\
\text { Total effective rate } \\
\text { of control group is } \\
78.80 \% .\end{array}$ & $\begin{array}{c}\text { Chinese } \\
\text { Academic } \\
\text { Journals }\end{array}$ \\
\hline 13 & $\begin{array}{c}\text { Sun et al. } \\
{[30]}\end{array}$ & 50 & $\begin{array}{c}71.4 \\
\text { years old }\end{array}$ & $\begin{array}{l}\text {-Experimental group } \\
\text { received acupressure. } \\
\text {-Control group received } \\
\text { slight touch }\end{array}$ & $\begin{array}{c}\text {-AIS } \\
\text { - PSQI }\end{array}$ & $\begin{array}{l}\text { Experimental } \\
\text { group }>\text { Control } \\
\text { group }\end{array}$ & $\begin{array}{l}\text { Chinese } \\
\text { Electronic } \\
\text { Periodical } \\
\text { Services }\end{array}$ \\
\hline 14 & $\begin{array}{c}\text { Lu et al. } \\
\text { [23] }\end{array}$ & 60 & 69.60 years old & $\begin{array}{l}\text {-Experimental group } \\
\text { received acupressure. } \\
\text {-Control group received } \\
\text { routine care }\end{array}$ & \multirow{2}{*}{$\begin{array}{l}- \text { PSQI } \\
-P F S \\
-P S Q I \\
-B D I\end{array}$} & \multirow{2}{*}{$\begin{array}{l}\text { Experimental } \\
\text { group> Control } \\
\text { group } \\
\text { Acupressure group } \\
\text { and TEAS group> } \\
\text { Control group } \\
\text { No significant } \\
\text { difference between } \\
\text { acupressure and } \\
\text { TEAS groups }\end{array}$} & \multirow[b]{2}{*}{$\begin{array}{c}\text { Cochrane } \\
\text { Library } \\
\text { PubMed }\end{array}$} \\
\hline 15 & $\begin{array}{l}\text { Tsay } \\
{[31]}\end{array}$ & 106 & 58.2 years old & $\begin{array}{c}\text {-Acupressure group received } \\
\text { acupressure } \\
\text {-TEAS group received teas } \\
\text { treatment } \\
\text {-Control groups only } \\
\text { received routine care }\end{array}$ & & & \\
\hline
\end{tabular}

Note: RCT, Randomized Controlled Trail; CT, Controlled Trail; PSQI, Pittsburgh Sleep Quality Index; SMHSQ St.Mary's Hospital Sleep Questionnaire; MMSE, mini-mental state examination; GHQ, General Health Questionnaire; STAI, State-Trait Anxiety Inventory; MLT, Urinary Melatonin; MRS, Menopause Rating Scale; AIS, Athens Insomnia Scale; MOS SF36, Medical Outcome Study Short Form 36. TEAS, Transcutaneous Electrical Acupoint Stimulation; PFS, Piper Fatigue Scale; BDI, Beck Depression Inventory.

\section{Effectiveness}

Basic information of the 15 reviewed studies was summarized in Table 2. Totally, there were 1134 insomnia patients included in trails with the average age of 63.76 years old. All the trails indicated that acupressure has a good therapeutic effectiveness on insomnia in the elderly.

Two-arm clinical trail: A two-arm trail was adopted by 9 of the studies (study $3,4,5,8,10,11,12,13,14$ ) to compare the outcome measures between or within two groups. Results showed that compare to the control groups which received routine care such as psychological health guidance, slight touch and sleep knowledge education, treatment groups that received acupressure except usual care were reported to be more effective (study $3,4,8,10,13,14$ ). To note that study 5 conducted by [19] set up a placebo group instead of a control group and use a wrist band device instead of traditional finger pressure. Although a good treatment effect has been received in the acupressure group, whether the placebo group was effective or not remains unknown. Study 11 and 12 of [20] and [19] compared the effectiveness between acupressure therapy and medication therapy according to the Sleeping duration time before and after treatment within each group. Results indicated that both acupressure and medications have therapeutic effect on insomnia but acupressure was found to be more effective. However, these two Chinese studies have not adopt common western standardized outcome measures such as Pittsburgh Sleep Quality Index (PSQI) and only present result by effective rate. Although the effective rate definition was not standardized [18], this expression straightforwardly presented the improvement or reduction in treatment effectiveness.

Three arm clinical trial: Other 6 studies adopted threearm trail design by setting three groups for better comparison. Except the acupressure group and control group, studies 1, $6,7,9$ added a sham acupressure group by applying pressure on non acupoints about $1-10 \mathrm{~cm}$ away from true acupoints avoiding meridians. Two of the trails (study 1 and 9) reported that acupressure was more effective than both sham group and control group while there was no significant difference between sham and control groups. However, results of study 6 and 7 indicated that sham acupressure was more effective than usual care in control group while acupressure treatment still have the highest effectiveness. The therapeutic effect in sham group 
may because the sham points were too close to the true points that still within the effective area. Physiological or psychological effect also works. Besides, the acupressure techniques used on non acupoints in sham groups may achieve body relaxation which also leads to a better sleep quality $[15,21]$. Another two studies ( 2 \& 15) compared the effectiveness between the mono therapy of acupressure and the combination therapy of Valerian acupressure or Transcutaneous Electrical Acupoints Stimulation (TEAS). It was reported that both the acupressure mono therapy and combination therapy no matter acupressure with valerian oil or acupressure with electrical stimulation had significant effectiveness in treating insomnia in the elderly when compared to control groups.

\section{Characteristics}

Acupressure treatment protocol details for insomnia of all the 15 studies were summarized in Table 3 regarding acupoints, pressure and specific interventions.

Table 3: Acupressure treatment protocol details of the 15 trails.

\begin{tabular}{|c|c|c|c|c|}
\hline 1 & $\begin{array}{l}\text { Chen et al. } \\
{[24]}\end{array}$ & $\begin{array}{l}\text { DU20 Baihui, GB20 Fengchi, } \\
\text { Ex-HN18Anmian, HT7 Shenmen (ear \& } \\
\text { hand) }\end{array}$ & 3 weeks & $\begin{array}{l}\text { About } 15 \text { minutes, } 2 \text { minutes for each acupoint,for } 5 \text { days per } \\
\text { week (rest on weekends) } \\
\text { The mean forces of fingers of left and right hands were from } \\
3.69 \text { to } 3.98 \mathrm{~kg} \\
\text { Interventions conducted between } 1 \mathrm{PM} \text { and prior to sleep } \\
\text { (before } 10 \mathrm{PM} \text { ) }\end{array}$ \\
\hline 2 & $\begin{array}{l}\text { Nesami et } \\
\text { al. } \\
{[27]}\end{array}$ & $\begin{array}{l}\text { HT7 Shenmen (ear \& hand); EX-HN3 } \\
\text { Yingtang, GB20 Fengchi,K11 Yongquan }\end{array}$ & 3 nights & $\begin{array}{l}\text { Totally } 18 \text { minutes, } 2 \text {-minute pressure for every point with a } \\
\text { 1-second rest rotationally at } 2 \text { rounds/sec with the thumb. } \\
\text { Intervention conducted three consecutive nights between } 7 \\
\text { PM and } 10 \mathrm{PM.} \\
\text { Received acupressure with a pressure of approximately } 3-4 \mathrm{~kg} \\
\text { in every point }\end{array}$ \\
\hline 3 & $\begin{array}{l}\text { Lei et al. } \\
{[26]}\end{array}$ & $\begin{array}{l}\text { Ex-HN18 Anmian, GB } 20 \text { Fengchi, } \\
\text { EX-HN } 5 \text { Taiyang, HT } 7 \text { Shenmen, } \\
\text { PC } 6 \text { Neiguan, SP } 6 \text { Sanyinjiao }\end{array}$ & 3 months & $\begin{array}{l}\text { Pressing each points each time lasting for } 1-2 \mathrm{~s} \text { and repeated } \\
40-50 \text { times. } \\
\text { There were } 4 \text { times massage every day, respectively at } 8: 00 \text {, } \\
12: 00,18: 00 \text { and the time before going to bed. }\end{array}$ \\
\hline 4 & $\begin{array}{l}\text { Shariati et } \\
\text { al. } \\
{[28]}\end{array}$ & $\begin{array}{l}\text { HT } 7 \text { Shenmen, LI4 Hegu, } \\
\text { SP } 6 \text { Sanyinjiao }\end{array}$ & 4 weeks & $\begin{array}{l}\text { The time of interventions was limited to } 15 \text { min, consisting of } \\
9 \text { min of acupoints massage ( } 3 \text { min per acupoint) and } 6 \text { min } \\
\text { of massage the areas near the acupionts to relax the person. } \\
3 \text { days per week. This action was done rapidly at the rate of } 2 \\
\text { rotations/s. }\end{array}$ \\
\hline 5 & $\begin{array}{l}\text { Nordio \& } \\
\text { Romanelli } \\
{[19]}\end{array}$ & HT7 Shenmen & 20 nights & $\begin{array}{l}\text { Wear devices }>\text { wrist band }>\text { for entire } 20 \text { nights period and at } \\
\text { exactly 22:00 h each night. }\end{array}$ \\
\hline 6 & $\begin{array}{l}\text { Tsay } \\
{[21]}\end{array}$ & $\begin{array}{l}\text { HT7 Shenmen (ear \& hand), } \\
\text { K11 Yongquan }\end{array}$ & 4 weeks & $\begin{array}{l}\text { The time of interventions was limited to } 14 \text { minutes,consisting } \\
\text { of } 5 \text { minutes of massage to relax the personand } 9 \text { minutes of } \\
\text { acupoints massage ( } 3 \text { minutes per acupoint). } \\
\text { force of finger pressure between } 3 \text { and } 4 \mathrm{~kg}\end{array}$ \\
\hline 7 & $\begin{array}{l}\text { Abedian et } \\
\text { al. } \\
{[15]}\end{array}$ & $\begin{array}{l}\text { HT7 Shenmen, SP } 6 \text { Sanyinjiao, } \\
\text { EX-HN3 Yingtang, GB20 Fengchi }\end{array}$ & 4 weeks & $\begin{array}{l}\text { The intervention time was limited to } 10 \text { minutes. It was done } \\
1 \text { to } 2 \text { hours before sleeping, each night (except Fridays) by } \\
\text { circular massage covering } 1 \mathrm{~cm} \text { diameter. } \\
\text { full pressure of approximately } 3-4 \mathrm{~kg} \text { on the points without } \\
\text { pain. }\end{array}$ \\
\hline 8 & $\begin{array}{l}\text { Zheng et al. } \\
\text { [32] }\end{array}$ & HT7 Shenmen, KI3 Taixi & 4 weeks & $\begin{array}{l}\text { The acupressure was applied } 5 \text { min per point once or twice per } \\
\text { day (before the noon break and night sleep, a total of } 40 \mathrm{~min} \\
\text { per day of acupressure). }\end{array}$ \\
\hline 9 & $\begin{array}{l}\text { Reza, et al. } \\
{[7]}\end{array}$ & $\begin{array}{l}\text { PC } 6 \text { Neiguan,K11 Yungchuan, } \\
\text { HT7 Shenmen (ear \& hand), } \\
\text { SP6Sanyinjiao, Ex-HN18 Anmian }\end{array}$ & 4weeks & $\begin{array}{l}\text { Subjects in the experimental group received acupressure } \\
\text { massage three times a week for } 4 \text { weeks; in addition, patients } \\
\text { were requested to efrain from massaging any acupoints during } \\
\text { the study period. The mean forces of fingers of left and } \\
\text { right hands were from } 3.39 \text { to } 3.21 \mathrm{~kg}\end{array}$ \\
\hline 10 & $\begin{array}{l}\text { Sun et al. } \\
{[22]}\end{array}$ & Shenmen point (HT7) & 5 weeks & $\begin{array}{l}\text { On both wrists with an interval of five-second pressure } \\
\text { followed by one-second rest for } 5 \text { min. The exerted pressure } \\
\text { should have been within } 3-5 \mathrm{~kg} \text {, using a standard scale. }\end{array}$ \\
\hline
\end{tabular}




\section{Global Journal of Intellectual \& Developmental Disabilities}

\begin{tabular}{|l|l|l|l|l|}
\hline 11 & $\begin{array}{l}\text { Qiu et al. } \\
{[20]}\end{array}$ & $\begin{array}{l}\text { DU23Shangxing,GB20 Fengchi, } \\
\text { EX-HN3 Yingtang,BL2 Zanzhu } \\
\text { EX-HN5 Taiyang, DU20 Baihui; SJ23 } \\
\text { Sizhukong, GB21 Jianjing }\end{array}$ & 26 days & $\begin{array}{l}\text { Apply pressure on Tai yang, Fengchi, Jian jin 10 times and other } \\
\text { acupoints 36 times at night }\end{array}$ \\
\hline 12 & $\begin{array}{l}\text { He et al. } \\
{[24]}\end{array}$ & $\begin{array}{l}\text { DU23 Shangxing, GB20 Fengchi, } \\
\text { EX-HN3 Yingtang, BL2 Zanzhu } \\
\text { EX-HN5 Taiyang, DU20 Baihui;, SJ23 } \\
\text { Sizhukong, GB21 Jianjing }\end{array}$ & 2 weeks & $\begin{array}{l}\text { Apply pressure on Tai yang, Fengchi, Jian jin 10 times and other } \\
\text { acupoints 36 times at night, last 20 mins }\end{array}$ \\
\hline 13 & $\begin{array}{l}\text { Sun et al. } \\
{[30]}\end{array}$ & $\begin{array}{l}\text { K11 Yongquan } \\
\text { S1 }\end{array}$ & 3 weeks & $\begin{array}{l}\text { The pressure is about 3-5 kg lasting 5min one time, limit to } \\
15 \text { min }\end{array}$ \\
\hline
\end{tabular}

Acupoints: Totally, there were 18 core acupoints noted in these 15 acupressure trials with the frequency shown in Figure 2. The most commonly used acupoint is the HT 7 (Shenmen) in hand with the frequency of 11 followed by GB 20 (Baihui) on head and K11 (Yongquan) on feet noted by 6 trials respectively. Besides, $50 \%$ of the 18 acupoints concentrating on head including DU20 (Baihui), DU23 (Shangxing), GB20 (Fengchi), BL2 (Zanzhu), SJ 23(Sizhukong), EX-HN5(Taiyang), EX-HN3(Yintang), Ex-HN18 (Anmian) and auricular HT 7 (Shenmen).

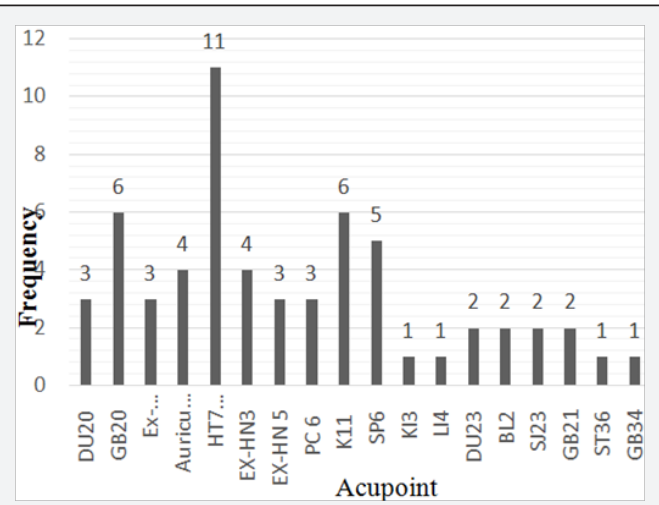

Figure 2: Acupoints frequency.

Pressure: A pressure need to be applied on each acupoint with a specific force range when conducting the acupressure intervention on human body. Too light pressure may have no or little effectiveness on insomnia which was usually used in sham group and have been proved to have no or little effectiveness on insomnia [22]. Otherwise, too much pressure may cause discomfort or even make patients injured with bruising. According to the intervention information in Table 3, the range of pressure force was usually between $3 \mathrm{~kg}$ to $5 \mathrm{~kg}$. To explore the most appropriate pressure force for various patients, the force could be gradually increased from $3 \mathrm{~kg}$ until the participant has a tolerable sensation of soreness [23].

Intervention: Most of the trials stated detailed interventions as shown in Table 3. To summarize, the mean duration of acupressure conduction lasted about $15 \mathrm{~min}$ one time and 1 to 4 times per day. Each acupoint applied pressure for 2 to $5 \mathrm{~min}$ with the frequency of 2-5 seconds pressure (2-3 rotations/s) and 1 second rest. Besides, many studies (study 1, 2, 5, 7, 8) suggested that it was better for patients to receive acupressure in the afternoon before noon break or in the evening prior to night sleep. Moreover, treatment period noted in the 15 trials ranged from 3 nights to 3 months. Most of the studies conducted interventions for 4 consecutive weeks [24-32].

\section{Conclusion}

This systematic review evaluated the effectiveness of acupressure for insomnia in the elderly and summarized the treatment characteristics of this tradition Chinese medicine technique. Although all of the selected acupressure studies see a significant effectiveness on insomnia in the elderly, there still remain some limitations that need to pay attention to. First, researches on acupressure for insomnia are limited, a systematic review process could only find a few studies that have reference value. Second, some of the studies especially those written in Chinese have a relatively lower Jada score without blind trial design and withdraw description. Third, when recruit participants, the history of other interventions such as drugs have not been recorded that might have some impacts on the results. Besides, some studies have insufficient subjects for each separate subgroup analysis. Moreover, more than half of the studies have not conduct a three-arm trial including an acupressure group, a sham acupressure group and a control group to minimize bias. Last but not the least, it is difficult for the elderly or careers to find accurate true acupoints and conduct appropriate intervention. In some trials sham acupoints were set too close to true points that fail to have an effective comparison with true acupressure group.

Consider the effectiveness, characteristics and limitations of current researches on acupressure, recommendation could be given as future directions of acupressure therapy and relevant therapeutic garments development. We recommend that the 5 most effective acupoints including HT 7 (Shenmen) on both hands, GB 20 (Baihui) on head and K11 (Yongquan) on both feet could be regarded as design reference considering their body locations. According to the result that $50 \%$ of the core acupoints concentrating on head, relevant therapeutic garment such as head band, gorro could be designed. Before garment design, investigation on a variety of requirements referring to human comfort or device usability should be conducted via questionnaire 
or interview to satisfy requirements of users. To note that physical sensation of comfort on different part of the therapeutic garments should be tested and adjusted when people wear it during different locomotion or in different posture. Combination therapy of acupressure such as herb acupressure, electrical acupressure, and heat acupressure could be adopted in garments by smart textiles, metal yarns, mini battery, etc. matching the meridians or acupoints on human body. Moreover, acupressure force applied on each selected acupoint should be tested while patients are static or dynamic. When the therapeutic garment is developed, it is necessary to conduct a randomized control trial with three-arm design (acupressure group, sham acupressure group and control group) among suitable subjects to ensure the effectiveness. Sham acupoints could not be too close to the true point in order to avoid the effective area and main meridians. On the whole, acupressure therapeutic garment has the potential to provide promising methods for insomnia and highly improve the life quality of the elderly.

\section{Acknowledgement}

The author would like to thank the funding from the Central Research Grant (research student account RUDM) and also thank Dr. Ameersing Luximon for helping paper risk of bias assessment as one of the evaluators.

\section{References}

1. Roepke SK, Ancoli-Israel S (2010) Sleep disorders in the elderly. Indian Journal of Medical Research 131(2): 302.

2. Liu X, Liu L (2005) Sleep habits and insomnia in a sample of elderly persons in China. Sleep 28(12): 1579-1587.

3. Galimi R (2010) Insomnia in the elderly: An update and future challenges. Giornale di Gerontologia 58(4): 231-247.

4. Roszkowska J, Geraci SA (2010) Management of insomnia in the geriatric patient. Am J Med 123(12): 1087-1090.

5. Montgomery P, Shepard LD (2010) Reviews in Clinical Gerontology Insomnia in older people Insomnia in older people. Reviews in Clinical Gerontology Reviews in Clinical Gerontology Reviews in Clinical Gerontology 20(20): 205-218.

6. Tsou MT (2013) Prevalence and risk factors for insomnia in communitydwelling elderly in northern Taiwan. Journal of Clinical Gerontology and Geriatrics 4(3): 75-79.

7. Reza H, Kian N, Pouresmail Z, Masood K, Sadat Seyed Bagher M, et al. (2010) The effect of acupressure on quality of sleep in Iranian elderly nursing home residents. Complement Ther Clin Pract 16(2): 81-85.

8. Tel H (2013) Sleep quality and quality of life among the elderly people. Neurology Psychiatry and Brain Research 19(1): 48-52.

9. Avidan AY, Fries BE, James ML, Szafara KL, Wright GT, et al. (2005) Insomnia and hypnotic use, recorded in the minimum data set, as predictors of falls and hip fractures in Michigan nursing homes J Am Geriatr Soc 53(6): 955-962.

10. Bloom HG, Ahmed I, Alessi CA, Ancoli-Israel S, Buysse DJ, et al (2009) Evidence-based recommendations for the assessment and management of sleep disorders in older persons. J Am Geriatr Soc 57(5): 761-789.
11. Wolkove N (2007) In older people Review. 176(9): 1299-1304.

12. Navarro B, López-Torres J, Andrés F, Latorre JM, Montes MJ, et al. (2013) Validation of the insomnia in the elderly scale for the detection of insomnia in older adults. Geriatr Gerontol Int 13(3): 646-653.

13. Kamel NS, Gammack JK (2006) Insomnia in the Elderly: Cause, Approach, and Treatment. Am J Med 119(6): 463-469.

14. Cherniack EP (2006) The use of alternative medicine for the treatment of insomnia in the elderly COMPLEMENTARY AND ALTERNATIVE. Experimental Gerontology, pp. 21-30.

15. Abedian Z, Eskandari L, Abdi H, Ebrahimzadeh S (2015) The Effect of Acupressure on Sleep Quality in Menopausal Women: A Randomized Control Trial. Iran J Med Sci 40(4): 328-334.

16. Chan Kf, Ng P, Ng Ky (2006) The effects of an intervention group with the support of non-pharmacological Chinese medicine on older Chinese adults with insomnia: a pilot study. International Social Work 49(6): 791-803.

17. Valente S (2015) Evaluating and Managing Insomnia: Nonpharmacological Treatments. Journal of Sleep Disorders \& Therapy $4(2): 2-5$.

18. Yeung WF (2012) Acupressure, reflexology, and auricular acupressure for insomnia: A systematic review of randomized controlled trials. Sleep Medicine 13(8): 971-984.

19. Nordio M, Romanelli F (2008) Efficacy of wrists overnight compression (HT 7 point) on insomniacs: Possible role of melatonin? Minerva Med 99(6): 539-547.

20. Qiu RJ (2000) Observation of the Clinical Effects of Acupuncture Points in Head for Insomnia. NangFang Journal of Nursing 7(1): 4.

21. Tsay SL, Rong JR, Lin PF (2003) Acupoints massage in improving the quality of sleep and quality of life in patients with end-stage renal disease. J Adv Nurs 42(2): 134-142.

22. Sun JL, Sung MS, Huang MY, Cheng GC, Lin CC (2010) Effectiveness of acupressure for residents of long-term care facilities with insomnia: A randomized controlled trial. Int J Nurs Stud 47(7): 798-805.

23. Lu MJ, Lin ST, Chen KM, Tsang HY, Su SF (2013) Acupressure Improves Sleep Quality of Psychogeriatric Inpatients. Nurs Res 62(2): 130-137.

24. Chen ML, Lin LC, Wu SC, Lin JG (1999) The effectiveness of acupressure in improving the quality of sleep of institutionalized residents. J Gerontol A Biol Sci Med Sc 54(8): M389-M394.

25. Foley DJ, Monjan AA, Brown SL, Simonsick EM, Wallace RB et al. (1995) Sleep complaints among elderly persons: an epidemiologic study of three communities. Sleep 18(6): 425-432.

26. Lei XF, Chen XL, Lin JX, Ai-fang Bao, Xu-cai Tao (2015) Clinical study on acupoint massage in improving cognitive function and sleep quality of elderly patients with hypertension. J Acupunct Tuina Sci 13(3): 175179.

27. Bagheri-Nesami M, Gorji MA, Rezaie S, Pouresmail Z, Cherati JY (2015) Journal of Traditional and Complementary Medicine Effect of acupressure with valerian oil $2.5 \%$ on the quality and quantity of sleep in patients with acute coronary syndrome in a cardiac intensive care unit. J Tradit Complement Med 5(4): 241-247.

28. Shariati A, Jahani S, Hooshmand M, Khalili N (2014) The effect of acupressure on sleep quality in hemodialysis patients. Complement Ther Med 20(6): 417-423.

29. Sun JL, Sung MS, Huang MY, Cheng GC, Lin CC (2010) Effectiveness of acupressure for residents of long-term care facilities with insomnia: A 
randomized controlled trial. Int J Nurs Stud 47(7): 798-805.

30. Sun J (2005) Nursing Home Effects of Acupressure for Improving the Insomnia of the Residents. Journal of Health Management 3(2): 121132

31. Tsay S, Cho Y, Chen M (2004) Acupressure and Transcutaneous
Electrical Acupoint Stimulation in Improving Fatigue, Sleep Quality and Depression in Hemodialysis Patients. Am J Chin Med 32(3): 407-416.

32. Zheng L, Yao Chen, Feng Chen, Ping Zhang, Li-Fang Wu (2014) Effect of acupressure on sleep quality of middle-aged and elderly patients with hypertension. International Journal of Nursing Sciences 1(4): 334-338.

\section{Your next submission with Juniper Publishers} will reach you the below assets

- Quality Editorial service

- Swift Peer Review

- Reprints availability

- E-prints Service

- Manuscript Podcast for convenient understanding

- Global attainment for your research

- Manuscript accessibility in different formats ( Pdf, E-pub, Full Text, Audio)

- Unceasing customer service

Track the below URL for one-step submission https://juniperpublishers.com/online-submission.php 